\title{
Efficacy and safety of selective internal radiation therapy with yttrium-90 for the treatment of unresectable hepatocellular carcinoma
}

\author{
Nguyen Van Thai ${ }^{1}$, Nguyen Tien Thinh ${ }^{1 *}$, Thai Doan Ky ${ }^{1}$, Mai Hong Bang ${ }^{1}$, Dinh Truong Giang ${ }^{1}$, Le Ngoc Ha ${ }^{2}$, \\ Mai Hong Son ${ }^{2}$, Dao Duc Tien ${ }^{3}$ and Hyun Woong Lee ${ }^{4^{*}}$
}

\begin{abstract}
Background: This retrospective analysis was undertaken to evaluate the efficiency of SIRT with Y-90 microspheres and determined prognostic factors affecting patients with unresectable HCC.

Methods: A total of 97 patients diagnosed with unresectable HCC who underwent SIRT with Y-90 microspheres. Patient survival was assessed using the Kaplan-Meier method, and prognostic factors affecting survival were assessed using log-rank tests and Cox proportional hazards regression.

Results: Among the 97 patients (90 males, mean age 60.4 12.3 years) who underwent SIRT, the median clinical follow-up was 16.4 (1.8-62) months. The median overall survival (OS) was $23.9 \pm 2.4$ months. Tumor response according to the Modified RECIST in patients followed up beyond 6 months included a complete response (CR) to treatment in 12 patients (18.8\%), partial response (PR) in 23 (35.8\%), stable disease (SD) in 8 (12.5\%), and progressive disease (PD) in 21 (32.8\%). Factors associated with longer OS included age $>65$ years, BCLC stage B, tumor size $<5 \mathrm{~cm}$, tumor burden $<25 \%$, and tumor response (CR/PR). In multivariate analysis, unilobar disease and objective tumor response (CR/PR) were predictors of longer OS.
\end{abstract}

Conclusion: SIRT was an effective treatment for unresectable HCC. Unilobar disease before SIRT and tumor response (CR/PR) were positive prognostic factors.

Keywords: Hepatocellular carcinoma, Selective internal radiation therapy, Yttrium-90, Survival, Tumor response

\section{Introduction}

Hepatocellular carcinoma (HCC) is the most common primary liver cancer. Worldwide, liver cancers are the fourth most common cause of cancer-related deaths.

\footnotetext{
*Correspondence: nguyentienthinha3108@yahoo.com.vn; Ihwdoc@yuhs.ac 1 Department of Hepato-Gastroenterology, 108 Military Central Hospital, 1 Tran Hung Dao Street, Hai Ba Trung District, Hanoi, Vietnam

${ }^{4}$ Department of Internal Medicine, Gangnam Severance Hospital, Yonsei University College of Medicine, 211 Eonju-ro, Gangnam-gu, Seoul 06273, Korea

Full list of author information is available at the end of the article
}

Based on annual projections, the World Health Organization estimates that more than 1 million patients will die from liver cancer in 2030. In the United States, the proportion of deaths owing to liver cancer increased by $43 \%$, from 7.2 to 10.3 deaths per 100,000 , between 2000 and 2016, with a 5-year survival rate of $18 \%$ [1]. However, only $20-30 \%$ of HCC patients are diagnosed at an early stage; most $(>70 \%)$ patients are diagnosed with unresectable disease and have poor overall prognosis [2]. Radical therapies, including resection or transplantation, are the gold standard for early-stage HCC [3]. Locoregional 
therapies such as transarterial embolotherapies, including conventional transarterial chemoembolization (TACE), bland transarterial embolization, and drugeluting bead TACE (DEB-TACE) have played an increasingly important role for patients with unresectable HCC. Targeted molecular therapies also have a recognized role, with sorafenib (regorafenib and lenvatinib) showing improved survival in advanced HCC patients [4]. SIRT with yttrium-90 (Y-90) microspheres is another feasible treatment option for this patient group, with a disease control rate of approximately $80 \%$ [5]. SIRT is usually indicated for intermediate- or advanced-stage patients who are poor candidates for TACE because of massive tumors, bilobar disease, or portal vein thrombosis (PVT). SIRT is also the treatment of choice for patients who are slightly above the criteria for curative treatments and who require tumor down-staging [6]. In recent years, SIRT with Y-90 microspheres has been proposed as an alternative to TACE. Several reports have shown that compared with TACE, SIRT is safe, with a lower rate of embolic complications such as fever and abdominal pain. SIRT is a recommended treatment for advanced HCC patients with PVT and has been shown to be efficacious, with comparable tumor response and survival rates to those of TACE [6]. This study evaluated the efficacy and safety of SIRT for unresectable HCC patients and analyzed prognostic factors affecting overall survival (OS) in this group of patients.

\section{Patients and methods}

\section{Patients}

We enrolled 97 unresectable HCC patients who underwent SIRT with Y-90 microspheres (SIR-spheres, Sirtex Medical, Sydney, Australia) at a single institution between October 2013 and March 2019. Starting in October 2013, all consecutive patients with HCC who were unsuitable for radical treatments (surgery, liver transplantation, or percutaneous ablation) or chemoembolization as a result of the presence of PVT or extensive tumor burden were assessed for SIRT. The inclusion criteria were patients diagnosed with $\mathrm{HCC}$ not amenable to curative surgical resection; at least 18 years of age; Eastern Cooperative Oncology Group (ECOG) performance status 0-2; Child-Pugh score $\leq 7$; life expectancy $>3$ months; without extrahepatic tumor burden; arteriovenous lung shunting $<20 \%$ in 99 mTc-macroaggregated albumin (MAA); and adequate hematology (granulocyte count $\geq 1.5 \times 10^{9} / \mathrm{L}$, platelets $\geq 50 \times 10^{9} / \mathrm{L}$ ), renal function (creatinine level $\leq 176.7 \mu \mathrm{mol} / \mathrm{L}$ ), hepatic function (bilirubin level $\leq 2 \mathrm{mg} / \mathrm{dL}$ ), and transaminase levels $<5$ times the institutional upper limit of the normal range. All patients provided informed consent. The exclusion criteria were infiltrative tumor type; tumor volume $>75 \%$ of the target liver volume or tumor nodules too numerous to count; tumor volume $>50 \%$ combined with an albumin level $<3.0 \mathrm{mg} / \mathrm{dL}$; ascites or clinical liver failure; pre-assessment angiogram showing abnormal vascular anatomy that would result in significant reflux of hepatic arterial blood to the stomach, pancreas, or bowel; disseminated extrahepatic malignant disease; prior external beam radiotherapy involving the liver; female patients who were pregnant or lactating; and severe comorbidities.

The study protocol was performed according to the principles of the 1975 Declaration of Helsinki and the study was approved by the Institutional Review Board of 108 Military Central Hospital (No;3761/HDDD). Informed consent was obtained from all individual participants included in the study.

\section{Methods}

The study was designed as a retrospective investigation. The radioembolization agent was Y-90 Resin Microspheres (SIR-Spheres ${ }^{\circledR}$, Sirtex Medical Limited, Sydney, Australia) made of $20-60-\mu \mathrm{m}$-sized resin or polymer beads with a high-energy, beta-emitting isotope without primary gamma emission [7].

Y-90 resin SIRT protocol: patients underwent comprehensive pretreatment evaluations comprising medical history, physical examination, and laboratory and imaging workup to confirm treatment eligibility. Diagnostic angiography accompanied by $99 \mathrm{mTc}-\mathrm{MAA}$ scintigraphy was then performed to identify vascular anatomy, HCC feeding vessels, aberrant vessels and extrahepatic collateral vessels feeding extrahepatic organs (especially the gastrointestinal tract), and the presence of intrahepatic or intratumoral arterioportal shunting; and, in case of PVT, the presence of bypassing blood flow through collateral vessels. Aberrant hepatic vessels and extrahepatic collaterals were coilembolized to prevent the inadvertent misplacement of $90 \mathrm{Y}$ resin microspheres into the gastrointestinal tract or pancreas. $99 \mathrm{mTc}-\mathrm{MAA}$ particles were then injected with the delivery catheter in the intended position for $90 Y$ resin microsphere infusion. Single-photon emission CT (SPECT) images were acquired to evaluate the 3D distributions of the microspheres inside the tumor and surrounding liver. The scintigraphy studies were usually performed within $1 \mathrm{~h}$ after 99mTc-MAA injection to assess pulmonary shunt and any unintended flow to other extrahepatic organs. Any patient who exhibited an intense mismatch between intrahepatic 99mTc-MAA distribution and liver lesions as viewed on fusion imaging with SPECT and CT was ineligible for treatment. The doses of resin microsphere treatments were calculated to maximize the therapeutic activity to 
tumorous tissue and minimize exposure of nontumoral parenchyma and lung tissue. Within 1 week after MAA angiography, SIR-Spheres ${ }^{\circledR}$ were administered following the same route used for MAA. Treatment protocol was approved institutionally.

Data collection and follow-up: the following data were collected at baseline: age, sex, presence of cirrhosis, PVT (segmental, branch, or main) and liver disease involvement (left lobe, right lobe, both lobes), extent of hepatic involvement (number and size of nodules) via CT, laboratory parameters including complete blood cell count, liver function tests, serum creatinine, Anti-HCV antibodies, hepatitis B surface antigen, ECOG, BCLC stages and Child-Pugh scores. Adverse events were classified for according to Common Terminology Criteria for Adverse Events, version 3.0. Tumor response following SIRT was assessed at 3 and 6 months by CT scan. In the evaluation of tumor response, the parenchymal and intravascular components of disease were assessed. With regards to changes in size of target lesions assessed according to mRECIST (8) in those patients with measurable disease, and the development of new lesions was assessed in all patients. If patients had PVT, changes in the extent of PVT were evaluated and classified as partial response (clearance or regression of tumor thrombus into a more distal portal vein segment), stable disease (no changes), or progressive disease (progression of tumor thrombus into a more proximal portal vein segment). Eventually, overall response was established as progressive disease whenever a patient had tumor progression in any of the three individual parameters (measurable target lesions, new lesions, or tumor thrombus) and controlled disease in any other case. Survival was determined from the time of treatment until death. Patients lost to followup were censored at the date of their last known visit.

\section{Statistical analysis}

Descriptive statistics for nominal, ordinal, and continuous variables, including frequency, median, and average, were used as appropriate. Proportions were compared using chi-squared tests with continuity correction or Fisher's exact tests, as appropriate. Survival was calculated from the day of treatment using the Kaplan-Meier method, and subgroup comparisons were performed using log-rank tests. We assessed the predictors of survival using univariate and multivariate Cox proportional hazard regression analyses. To assess the variation in each liver function test over the previous follow-up, we used one-way analysis of variance. The analysis was performed using IBM SPSS Statistics for Windows, version 20.0 (SPSS GmbH, Munich, Germany). Significant differences were defined as $\mathrm{p}<0.05$.

\section{Results}

Patient characteristics at baseline

Overall, 97 patients with $\mathrm{HCC}$ underwent treatment with Y-90 microspheres with the median follow-up period for all patients after SIRT was 16.4 months (range 1.862 months). Ten patients were lost to follow-up within 3 months after TARE. Twenty-three patients who had non-measurable parenchymal target disease (diffuse or massive tumors) did not perform computerized tomography at 6 months after TARE. It was impossible to evaluate for tumor response.

The mean age was $60.4 \pm 12.3$ (range 25-89) years; 90 patients were male $(92.8 \%)$ and about half of them were positive for hepatitis B. Most patients had ECOG 1 (73.2\%); $19.6 \%$ received prior treatments, including resection, radiofrequency ablation, percutaneous ethanol injection therapy, and TACE. Ninety-one patients (93.8\%) had a Child-Pugh score of A. Barcelona Clinic Liver Cancer (BCLC) stage $C(n=59,60.8 \%)$ was more common than BCLC B $(\mathrm{n}=38,39.2 \%$ and 61 patients (62.9\%) had portal vein invasion. The mean tumor size was $10.2 \pm 3.5 \mathrm{~cm}$. The disease was limited to one lobe in 81 patients, and 16 patients had bilobar disease (Table 1). The mean dose of Y-90 was $1.6 \pm 0.6 \mathrm{GBq}$, with an estimated $5.2 \pm 3.6 \%$ being shunted to the lungs.

\section{Treatment response}

Among all patients, 64 were followed up beyond 6 months after radioembolization therapy and underwent follow-up CT based on which tumor response was assessed according to the RECIST guidelines. Complete response (CR) and partial response (PR) to treatment were observed in $12(18.8 \%)$ and 23 patients $(35.8 \%)$, respectively, while $8(12.5 \%)$ and 21 (32.8\%) patients, respectively, had stable disease (SD) and progressive disease (PD). The tumor diameter decreased from a mean of $10.2 \pm 3.5 \mathrm{~cm}$ at baseline to $6.9 \pm 3.3 \mathrm{~cm}$ at 6 months after treatment (Table 2).

The median survival after SIRT was 23.9 months, with the 6-, 12-, 24-, 36- and 48-month survival rates being $77.9 \%, 56.7 \%$, 39.2\%, 31\%, and $18.5 \%$, respectively (Fig. 1 ). Two patients had SD, with a survival of 48 months. 61 patients died $1-48$ months after SIRT. In 53 of the 61 patients who died, tumor progression was the suspected cause of death. Sites of progression included target lesions $(n=20)$, new lesions $(n=12)$, tumor thrombus $(n=9)$ and extrahepatic metastases $(n=12)$. Among the 64 patients who reached 6-month follow-up and were evaluable for response, 21 (32.8\%) had progressive disease. Sites of progression included new lesions $(n=19)$ and tumor thrombus $(\mathrm{n}=2)$.

Overall, univariate analysis of OS showed significant interrelations between age, tumor size, tumor 
Table 1 Baseline characteristics

\begin{tabular}{|c|c|}
\hline Variables & $\mathrm{N}=97(\%)$ \\
\hline \multicolumn{2}{|l|}{ Age, years } \\
\hline$<65$ & $61(62.9)$ \\
\hline$\geq 65$ & $36(37.1)$ \\
\hline \multicolumn{2}{|l|}{ Sex } \\
\hline Male & $90(92.8)$ \\
\hline Female & $7(7.2)$ \\
\hline \multicolumn{2}{|l|}{ Etiology } \\
\hline Hepatitis B & $54(55.7)$ \\
\hline Hepatitis C & $4(4.1)$ \\
\hline Alcohol & $3(3.1)$ \\
\hline Unknown & $36(37.1)$ \\
\hline \multicolumn{2}{|l|}{ Performance status } \\
\hline 0 & $14(14.4)$ \\
\hline 1 & $71(73.2)$ \\
\hline 2 & $12(12.4)$ \\
\hline \multicolumn{2}{|l|}{ Prior treatment } \\
\hline None & $78(80.4)$ \\
\hline Resection & $5(5.2)$ \\
\hline Radiofrequency ablation & $3(3.1)$ \\
\hline Percutaneous ethanol injection therapy & $2(2.1)$ \\
\hline Transarterial chemoembolization & $9(9.3)$ \\
\hline \multicolumn{2}{|l|}{ Method of diagnosis } \\
\hline Liver biopsy & $59(60.8)$ \\
\hline Imaging & $38(39.2)$ \\
\hline \multicolumn{2}{|l|}{ Pathology } \\
\hline Well-differentiated & $10(10.3)$ \\
\hline Moderate differentiated & $38(39.2)$ \\
\hline Poorly differentiated & $3(3.1)$ \\
\hline No defined & $8(8.2)$ \\
\hline \multicolumn{2}{|l|}{ Cirrhosis on imaging } \\
\hline Present & 94 (96.9) \\
\hline Absent & $3(3.1)$ \\
\hline \multicolumn{2}{|l|}{ Tumor distribution } \\
\hline Unilobar, right & $74(76.3)$ \\
\hline Unilobar, left & $7(7.2)$ \\
\hline Bilobar & $16(16.5)$ \\
\hline \multicolumn{2}{|l|}{ Portal vein invasion } \\
\hline None & $36(37.1)$ \\
\hline Below subsegmental & $1(1.0)$ \\
\hline Subsegmental & $28(28.9)$ \\
\hline Lobar & $25(25.8)$ \\
\hline Main & $7(7.2)$ \\
\hline \multicolumn{2}{|l|}{ Index lesion size (cm) } \\
\hline$<5$ & $6(6.2)$ \\
\hline $5-10$ & $40(41.2)$ \\
\hline$>10$ & $51(52.6)$ \\
\hline \multicolumn{2}{|l|}{ Tumor burden* (\%) } \\
\hline$<25$ & $44(45.4)$ \\
\hline $25-50$ & $38(39.2)$ \\
\hline
\end{tabular}

Table 1 (continued)

\begin{tabular}{lc}
\hline Variables & $\mathbf{N}=\mathbf{9 7}(\mathbf{\%})$ \\
\hline$>50$ & $9(9.3)$ \\
No defined & $6(6.2)$ \\
Alpha-fetoprotein & \\
$<100$ & $45(46.4)$ \\
$\geq 100$ & $52(53.6)$ \\
Total bilirubin (mg/dL) & \\
$<2$ & $93(95.9)$ \\
$2--3$ & $4(4.1)$ \\
$>3$ & $0(0)$ \\
Albumin (mg/dL) & \\
$>3.5$ & $83(85.6)$ \\
$2.8-3.5$ & $12(12.4)$ \\
$<2.8$ & $2(2.1)$ \\
Child-Pugh class & \\
A & $91(93.8)$ \\
B & $6(6.2)$ \\
C & $0(0)$ \\
BCLC ${ }^{+}$stage & \\
A & $0(0)$ \\
B & $38(39.2)$ \\
C & $59(60.8)$ \\
D & $0(0)$ \\
${ }^{*}$ Tumor burden, Tumor volume/liver volume & \\
${ }^{B}$ CLC, Barcelona Clinic Liver Cancer staging system &
\end{tabular}

burden, tumor distribution, BCLC stage, and radiological response to treatment. Survival did not differ significantly in subgroups of patients according to sex, ECOG score, prior treatment, portal vein invasion, alpha-fetoprotein (AFP) level, and Child-Pugh score (Table 3).

Significant differences were noted in terms of survival during 5 years follow-up between Kaplan-Meier survival curves of the two groups according to age, BCLC stage, tumor burden and objective tumor response (Fig. 2a-d), using the log-rank test $(p$ value $=0.017,0.018,<0.001$, and $<0.001$, respectively).

In multivariate analysis, the tumor distribution (hazard ratio [HR] for unilobar vs. bilobar; 0.10, confidential interval; 0.02-0.75, $p=0.012$ ) and best overall mRECIST response (HR for $\mathrm{CR} / \mathrm{PR}$ vs $\mathrm{SD} / \mathrm{PD}$; 0.09, confidential interval; 0.03-0.29, $p=0.001)$ were independent predictors of OS (Table 3 ).

\section{Clinical toxicity}

In total, 38 (39.2\%) patients developed clinical toxicity after treatment, which included abdominal pain $(\mathrm{n}=25$, $25.8 \%)$, fatigue $(n=19,19.6 \%)$, fever $(n=6,6.2 \%)$, and nausea/vomiting $(n=6,6.2 \%)$. These complications were 
Table 2 Tumor response based on mRECIST guidelines

\begin{tabular}{|c|c|c|c|c|c|c|}
\hline \multirow[t]{2}{*}{ Time point } & \multirow{2}{*}{$\begin{array}{l}\text { Lesion diameter }(\mathrm{cm}) \\
\text { mean } \pm \text { standard deviation }\end{array}$} & \multirow{2}{*}{$\begin{array}{l}\text { Number with available } \\
\text { data }(\mathrm{N}=97)\end{array}$} & \multicolumn{4}{|c|}{ mRECIST*, n (\%) } \\
\hline & & & CR & PR & SD & PD \\
\hline Baseline & $10.2 \pm 3.5$ & 97 & - & - & - & - \\
\hline 3 months & $8.2 \pm 3.5$ & 87 & $10(11.5)$ & $42(48.3)$ & $19(21.8)$ & $16(18.4)$ \\
\hline 6 months & $6.9 \pm 3.3$ & 64 & $12(18.8)$ & $23(35.9)$ & $8(12.5)$ & $21(32.8)$ \\
\hline
\end{tabular}

*mRECIST, Response Evaluation Criteria In Solid Tumors; CR, complete response; PR, partial response; SD, stable disease; PD, progressive disease

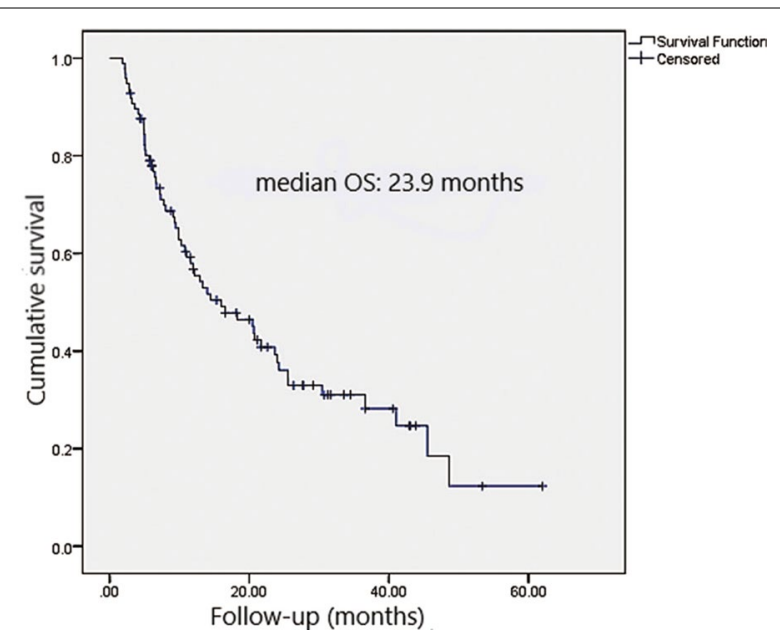

Fig. 1 Kaplan-Meier survival curves. (A) Median overall survival was 23.9 months. The median survival after SIRT was 23.9 months with 6-, $12-, 24-, 36-$, and 48 -month survival of $77.9 \%, 56.7 \%, 39.2 \%, 31 \%$, and $18.5 \%$, respectively

mild and resolved without active intervention. No patient died because of the treatment.

During follow-up, one patient experienced gastroduodenal ulceration, one experienced pneumonitis, and one experienced gastrointestinal (GI) bleeding 3 months after SIRT. Two patients developed GI bleeding after 6 months.

\section{Discussion}

For many HCC patients, curative surgical therapies (resection or transplantation) are not an option owing to tumor bulk or the presence of metastatic disease. Locoregional therapies such as SIRT with Y-90 are viable alternative methods of reducing tumor burden, prolonging OS, and improving quality of life. Our study demonstrated that SIRT administered to 97 patients with unresectable HCC resulted in a median survival of 23.9 months, with a 3 -year survival of $31 \%$. Our encouraging findings are concordant with those reported in earlier series. Salem et al. reported a median survival of 20.5 months and 3-year survival of $25 \%$ in 123 patients who underwent SIRT [8]. Mantry et al. reported a median survival of 13.1 months in 111 patients treated with SIRT [9]. In the ENRY study, a prospective European multicenter trial of Y-90 resin SIRT in 325 patients with unresectable HCC, the median OS was 12.8 months (95\% confidence interval [CI]: 10.9-15.7) [10]. However, other studies reported longer OS. For instance, Saxena et al. reported a median OS of 27.7 months and 3-year survival of $26 \%$ in 45 patients with unresectable HCC [6]. A meta-analysis of 21 published reports showed that the pooled OS was $63 \%$ and $27 \%$ at 1 - and 3-years respectively in intermediate-stage $\mathrm{HCC}$, whereas OS was $37 \%$ and $13 \%$ at 1 - and 3-years respectively in advanced HCC because of the presence of portal vein thrombosis [11].

In patients with advanced HCC, sorafenib has become the standard treatment after the SHARP trial showed a higher median OS for sorafenib (10.7 months) than for placebo (7.9 months) [12]. The SARAH trial demonstrated similar OS between patients treated with SIRT and sorafenib (8.54 vs. 10.58 months), however, a lower rate of serious adverse events was observed in the Y-90 arm $(27 \%)$ than in the sorafenib arm (>50\%). In addition, the tumor response rate was better in the Y-90 arm (16.5\%) than in the sorafenib arm (1.7\%) [7]. Collectively, these data support the role of SIRT rather than systemic agents in the management of unresectable $\mathrm{HCC}$, whenever possible.

The current recommended TACE is the standard of care and first-line treatment for intermediatestage unresectable HCC [13]. The median survival is around 20 months in most series, ranging from 14 to 45 months [14]. Several studies comparing the relative efficacy of SIRT and TACE showed that SIRT is as effective, if not more effective, in inducing a radiological response and improving survival than TACE [15]. Clinically, most of the disadvantages of TACE manifest as pain and post-embolization syndrome, often requiring hospitalization. Moreover, TACE is contraindicated in patients with main PVT because of its profound ischemic effects. Salem et al. compared the results of 122 patients who underwent SIRT with those of 123 patients treated with TACE [8]. Complications, particularly, abdominal pain and liver dysfunction were more common following TACE than 
Table 3 Significant predictors of survival

\begin{tabular}{|c|c|c|c|c|c|}
\hline \multirow[t]{2}{*}{ Variables } & & \multicolumn{2}{|c|}{ Univariate analysis } & \multicolumn{2}{|c|}{ Multivariate analysis } \\
\hline & & $\mathrm{HR}(95 \% \mathrm{Cl})^{*}$ & $p$ Value & $\mathrm{HR}(95 \% \mathrm{Cl})$ & $p$ Value \\
\hline \multirow[t]{2}{*}{ Age } & $<65$ & $1.97(1.12-3.46)$ & 0.017 & & \\
\hline & $\geq 65$ & 1 & & & \\
\hline \multirow[t]{2}{*}{ Sex } & Male & $1.25(0.45-3.49)$ & 0.661 & & \\
\hline & Female & 1 & & & \\
\hline \multirow[t]{2}{*}{ ECOG } & 0,1 & 1 & & & \\
\hline & 2 & $1.39(0.18-6.67)$ & 0.732 & & \\
\hline \multirow[t]{2}{*}{ Prior treatment } & No & $0.96(0.51-1.81)$ & 0.893 & & \\
\hline & Yes & 1 & & & \\
\hline \multirow[t]{2}{*}{ Tumor distribution } & Unilobar & $0.87(0.43-0.98)$ & 0.044 & $0.10(0.02-0.75)$ & 0.012 \\
\hline & Bilobar & 1 & & & \\
\hline \multirow[t]{2}{*}{ Portal vein invasion } & No & $0.61(0.35-1.06)$ & 0.083 & & \\
\hline & Yes & 1 & & & \\
\hline \multirow[t]{2}{*}{ Index lesion size (cm) } & $\leq 10$ & $0.39(0.22-0.67)$ & 0.001 & $0.92(0.26-3.21)$ & 0.672 \\
\hline & $>10$ & 1 & & & \\
\hline \multirow[t]{2}{*}{ Tumor burden ${ }^{\dagger}(\%)$} & $\leq 50$ & $0.18(0.07-0.43)$ & 0.001 & $0.94(0.58-9.52)$ & 0.733 \\
\hline & $50-75$ & 1 & & & \\
\hline \multirow[t]{2}{*}{ Alpha-fetoprotein } & $<100$ & $0.63(0.38-1.05)$ & 0.072 & & \\
\hline & $\geq 100$ & 1 & & & \\
\hline \multirow[t]{2}{*}{ Child-Pugh class } & Child A & 1 & & & \\
\hline & Child B & $1.27(0.40-4.06)$ & 0.691 & & \\
\hline \multirow[t]{2}{*}{ BCLC stage $e^{\ddagger}$} & $B C L C B$ & $0.48(0.26-0.89)$ & 0.018 & $0.43(0.12-1.15)$ & 0.317 \\
\hline & $\mathrm{BCLCC}$ & 1 & & & \\
\hline \multirow[t]{2}{*}{ Radiologic response $\left(\mathrm{mRECIST} \mathrm{T}^{5}\right)$} & $C R / P R$ & $0.14(0.06-0.32)$ & 0.001 & $0.09(0.03-0.29)$ & 0.001 \\
\hline & $\mathrm{SD} / \mathrm{PD}$ & 1 & & & \\
\hline
\end{tabular}

*HR, hazard ratio; $\mathrm{Cl}$, Confidential Index

${ }^{\dagger}$ Tumor burden, Tumor volume/liver volume

${ }^{\ddagger}$ BCLC, Barcelona Clinic Liver Cancer staging system

${ }^{\S}$ mRECIST, Response Evaluation Criteria In Solid Tumors; CR, complete response; PR, partial response; SD, stable disease; PD, progressive disease

following SIRT $(\mathrm{p}<0.05)$. A trend toward higher objective response rates ( 49 vs. $36 \%, p=0.1$ ) was observed in patients undergoing SIRT. The time-to-progression was longer following SIRT than following TACE (13.3 vs. 8.4 months, $p=0.046)$; however, the median OS did not differ significantly ( 20.5 vs. 17.4 months, $p=0.2$ ). Particularly, in meta-analysis of 10 published reports, SIRT showed a statistically significant benefit as compared to TACE in terms of higher progression-free survival rate, although SIRT and TACE showed similar overall survival [16]. These studies illustrated better quality of life outcomes in patients undergoing SIRT rather than those undergoing TACE, although more patients presented with advanced disease.

In the univariate analysis, the prognosticators of OS were age, tumor size, liver tumor burden, tumor distribution, BCLC stage, and radiological response to treatment. Patients older than 65 years fared better than their younger counterparts, likely because the proportion BCLC B patients older than 65 years was higher than that in younger patients $(55.6 \%$ vs. $36.1 \%, p=0.061)$. Considering recent reports, radioembolization appears to be a well-tolerated and effective treatment option for elderly patients without concomitant disease [8, 17]. In our study, the median OS was significantly better in patients with BCLC stage B than in those with stage $C$ (31.2 vs. 21.2 months; $p=0.018$ ). Salem et al., Ozkan et al., and Sangro et al. also emphasized the significant relationship between BCLC stage and survival $[8,10,18]$. Multivariate analysis in the present study showed that unilobar tumor distribution and tumor response were prognostic factors for OS. This finding was expected and has been reported 

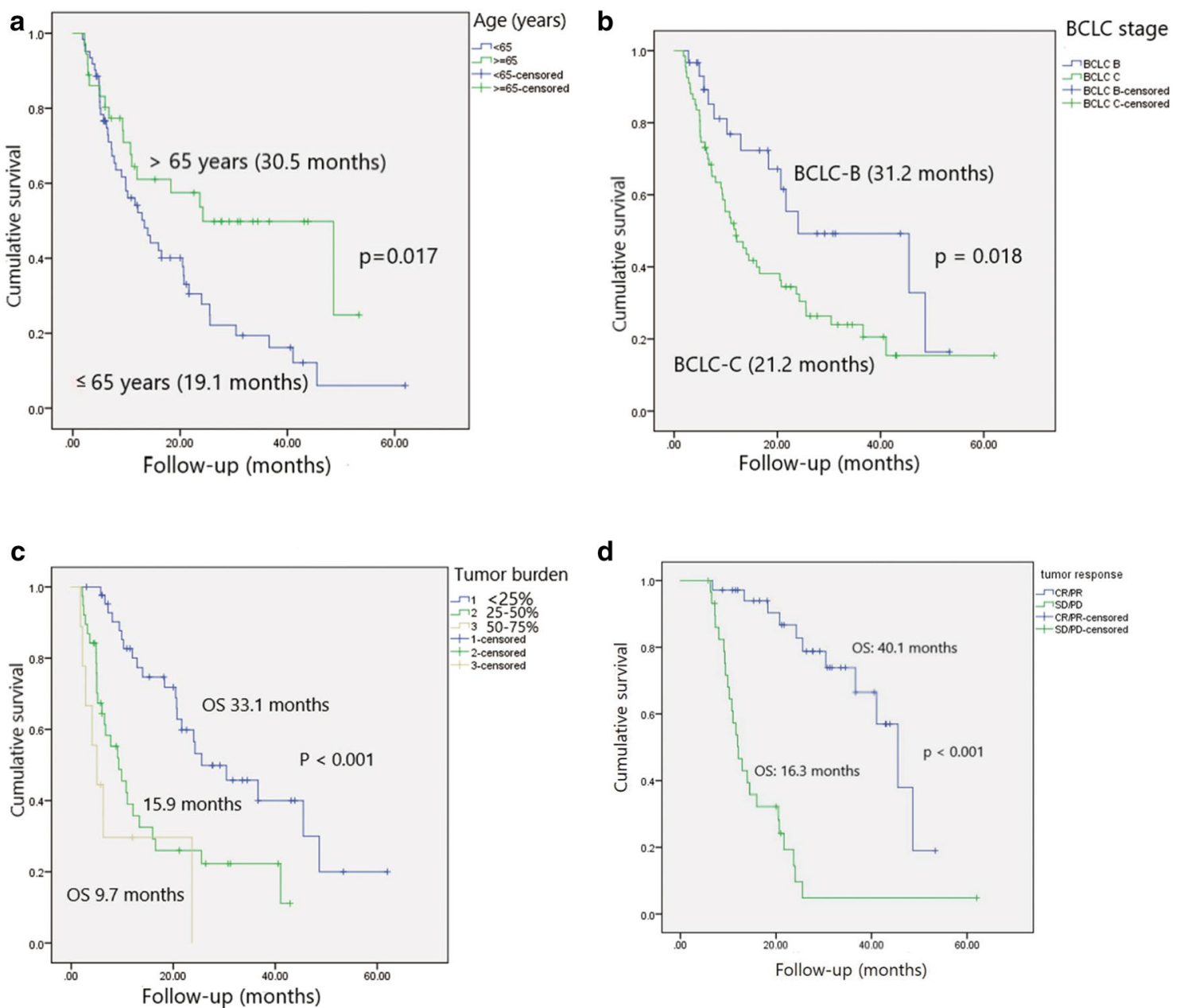

Fig. 2 Kaplan-Meier curves demonstrating survival based on age, BCLC stage, tumor burden, and tumor response. a The median overall survival is significantly better in patients aged above 65 years than in those aged below 65 years ( 30.5 vs. 19.1 months; $p=0.017$ ). b The median overall survival was significantly better in patients with BCLC stage B than in those with BCLC stage C (31.2 vs. 21.2 months; $p=0.018)$. $\mathbf{c}$ The median overall survival was significantly better in patients with tumor burden under $25 \%$ than in those with tumor burden $25-50 \%$ and above $50 \%$ (33.1, vs. 15.9 and 9.7 months; $p<0.001)$. d The median overall survival was significantly better in patients with objective tumor response (CR/PR) than in those with SD/PD (40.1 vs. 16.3 months; $p<0.001)$

previously. Mazzaferro et al. showed that radiological response $(\mathrm{CR} / \mathrm{PR} / \mathrm{SD}$ vs. $\mathrm{PD})$ was an independent risk factor for OS $(p=0.048)$ [19].

In this study, the clinical toxicity rate was $39.2 \%$, and all events, including abdominal pain, fatigue, fever, and nausea/vomiting, were self-limiting and resolved without active intervention. No treatment-related mortality was observed, similar to reports from earlier series[6, 8]. During the follow-up, one patient developed dyspnea 3 months after radioembolization, suggesting radiation-induced pneumonitis. One patient presented with gastroduodenal ulceration and one case had variceal esophagus bleeding 3 months after treatment. At 6 weeks after SIRT, two patients developed GI bleeding owing to hepatic failure. Mayer et al. reported about three patients with gastroduodenal ulcers, one with radiation pneumonitis, and one with septicemia [20].

Our study also has some limitations. First, the retrospective nature of this study and the relatively small sample size obviously limits the strength of evidence. 
Second, the median follow-up period for all patients was too short. In particular, the presence of a high percentage of patients for whom the tumor response could not be evaluated. It is expected to have affected these results. The hazard ratios of the tumor distribution and the best overall mRECIST response (CR/PR) were likely to be overestimated. It is another limitation for the results of this study. Third, we were not able to analyze the severity of non-laboratory adverse events because the description of the adverse events is not properly recorded. Finally, the Kaplan-Meier method is the most commonly used timeto-event analysis. However, since not all the patients died because of cancer, a survival method that considers the competing risk would be more appropriate.

\section{Conclusions}

Our study results add to the growing literature on SIRT for unresectable HCC, demonstrating that SIRT is safe and effective with high objective response rates (CR/PR 54,7\%) and median OS of 23.9 months. The unilobar disease before SIRT and tumor response (CR/PR) are significant predictors of survival.

\begin{abstract}
Abbreviations
BCLC: Barcelona clinic liver cancer; CR: Complete response; CT: Computed tomography; TACE: Transarterial chemoembolization; Gl: Gastrointestinal; HCC: Hepatocellular carcinoma; HR: Hazard ratio; MAA: 99MTc-macroaggregated albumin; mRECIST: Modified response evaluation criteria in solid tumor; OS: Overall survival; PD: Progressive disease; PR: Partial response; PVT: Portal vein thrombosis; SD: Stable disease; SIRT: Selective internal radiation therapy; SPECT: Single-photon emission computed tomography; Y-90: Yttrium-90.
\end{abstract}

\section{Authors' contributions}

NTT and TNV have full access to all data in the study and take the responsibility of data integrity and accuracy of analysis. Study concept and design: NTT, NVT, MHB, TDK. Acquisition of data: DDT, NVT, TDK, MHS, DTG, LNH. Analysis and interpretation of data: NVT, NTT and HWL. Validation: MHB, NTT, HWL. Drafting of the manuscript: TNV. Statistical analysis: TNV and HWL. Writing - review \& editing: NTT, HWL. All authors read and approved the final manuscript.

\section{Funding}

This study was supported by the Research Grant of Gangnam Severance Cancer Hospital and the Korean Liver Cancer Association (KLCA) International Fellowship Program. (No; KLCA-2019).

\section{Availability of data and materials}

The datasets generated and analyzed during the current study are available from the corresponding author on reasonable request.

\section{Declarations}

\section{Ethics approval and consent to participate}

The study protocol was performed according to the principles of the 1975 Declaration of Helsinki and the study was approved by the Institutional Review Board of 108 Military Central Hospital (No;3761/HDDD). Informed consent was obtained from all individual participants included in the study.

\section{Consent for publication}

Patients signed informed consent forms regarding the publication of their data.

\section{Competing interests}

The authors declare no conflict of interest about this work.

\section{Author details}

${ }^{1}$ Department of Hepato-Gastroenterology, 108 Military Central Hospital, 1 Tran Hung Dao Street, Hai Ba Trung District, Hanoi, Vietnam. ${ }^{2}$ Department of Nuclear Medicine, 108 Military Central Hospital, Hanoi, Vietnam. ${ }^{3}$ Department of Hepato-Gastroenterology, 175 Military Hospital, Ho Chi Minh, Vietnam. ${ }^{4}$ Department of Internal Medicine, Gangnam Severance Hospital, Yonsei University College of Medicine, 211 Eonju-ro, Gangnam-gu, Seoul 06273, Korea.

Received: 12 February 2021 Accepted: 30 April 2021

Published online: 12 May 2021

\section{References}

1. Villanueva A. Hepatocellular carcinoma. N Engl J Med. 2019;380(15):1450-62.

2. Wang EA, Stein JP, Bellavia RJ, Broadwell SR: Treatment options for unresectable HCC with a focus on SIRT with Yttrium-90 resin microspheres. Int J Clin Pract 2017, 71(11).

3. European Association for the Study of the Liver. Electronic address eee, European Association for the Study of the L: EASL Clinical Practice Guidelines: Management of hepatocellular carcinoma. J Hepatol. 2018;69(1):182-236

4. Guney IB. HCC locoregional therapies: Yttrium-90 (Y-90) selective internal radiation therapy (SIRT). J Gastrointest Cancer. 2017;48(3):276-80.

5. Sangro B, Inarrairaegui M, Bilbao Jl. Radioembolization for hepatocellular carcinoma. J Hepatol. 2012;56(2):464-73.

6. Saxena A, Meteling B, Kapoor J, Golani S, Danta M, Morris DL, Bester L. Yttrium-90 radioembolization is a safe and effective treatment for unresectable hepatocellular carcinoma: a single centre experience of 45 consecutive patients. Int J Surg. 2014;12(12):1403-8.

7. Yttrium Y 90. In: Drugs and Lactation Database (LactMed). edn. Bethesda (MD); 2006.

8. Salem R, Lewandowski RJ, Kulik L, Wang E, Riaz A, Ryu RK, Sato KT, Gupta $\mathrm{R}$, Nikolaidis $\mathrm{P}$, Miller FH, et al. Radioembolization results in longer time-to-progression and reduced toxicity compared with chemoembolization in patients with hepatocellular carcinoma. Gastroenterology. 2011;140(2):497-507.

9. Mantry PS, Mehta A, Madani B, Mejia A, Shahin I. Selective internal radiation therapy using yttrium-90 resin microspheres in patients with unresectable hepatocellular carcinoma: a retrospective study. J Gastrointest Oncol. 2017:8(5):799-807.

10. Sangro B, Carpanese L, Cianni R, Golfieri R, Gasparini D, Ezziddin S, Paprottka PM, Fiore F, Van Buskirk M, Bilbao Jl, et al. Survival after yttrium-90 resin microsphere radioembolization of hepatocellular carcinoma across Barcelona clinic liver cancer stages: a European evaluation. Hepatology. 2011;54(3):868-78.

11. Rognoni C, Ciani O, Sommariva S, Facciorusso A, Tarricone R, Bhoori S, Mazzaferro V. Trans-arterial radioembolization in intermediate-advanced hepatocellular carcinoma: systematic review and meta-analyses. Oncotarget. 2016;7(44):72343-55.

12. Llovet JM, Ricci S, Mazzaferro V, Hilgard P, Gane E, Blanc JF, de Oliveira AC, Santoro A, Raoul JL, Forner A, et al. Sorafenib in advanced hepatocellular carcinoma. N Engl J Med. 2008;359(4):378-90.

13. Forner A, Gilabert M, Bruix J, Raoul JL. Treatment of intermediate-stage hepatocellular carcinoma. Nat Rev Clin Oncol. 2014;11(9):525-35.

14. Llovet JM, Bruix J. Systematic review of randomized trials for unresectable hepatocellular carcinoma: Chemoembolization improves survival. Hepatology. 2003;37(2):429-42. 
15. Pitton MB, Kloeckner R, Ruckes C, Wirth GM, Eichhorn W, Worns MA, Weinmann A, Schreckenberger M, Galle PR, Otto G, et al. Randomized comparison of selective internal radiotherapy (SIRT) versus drug-eluting bead transarterial chemoembolization (DEB-TACE) for the treatment of hepatocellular carcinoma. Cardiovasc Intervent Radiol. 2015;38(2):352-60.

16. Facciorusso A, Serviddio G, Muscatiello N. Transarterial radioembolization vs chemoembolization for hepatocarcinoma patients: a systematic review and meta-analysis. World J Hepatol. 2016;8(18):770-8.

17. Golfieri R, Bilbao Jl, Carpanese L, Cianni R, Gasparini D, Ezziddin S, Paprottka PM, Fiore F, Cappelli A, Rodriguez M, et al. Comparison of the survival and tolerability of radioembolization in elderly vs. younger patients with unresectable hepatocellular carcinoma. J Hepatol. 2013:59(4):753-61.

18. Ozkan ZG, Poyanli A, Ucar A, Kuyumcu S, Akyuz F, Keskin S, Saglam S, Yilmaz E, Karaca C, Turkmen C. Favorable survival time provided with radioembolization in hepatocellular carcinoma patients with and without portal vein thrombosis. Cancer Biother Radiopharm. 2015;30(3):132-8.

19. Mazzaferro V, Sposito C, Bhoori S, Romito R, Chiesa C, Morosi C, Maccauro M, Marchiano A, Bongini M, Lanocita R, et al. Yttrium-90 radioembolization for intermediate-advanced hepatocellular carcinoma: a phase 2 study. Hepatology. 2013;57(5):1826-37.

20. Meyer C, Pieper CC, Ahmadzadehfar H, Lampe NA, Matuschek EME, Maschke TA, Enkirch SJ, Essler M, Spengler U, Schild HH. Yttrium-90 radioembolization of unresectable hepatocellular carcinoma - a single center experience. Onco Targets Ther. 2017;10:4773-85.

\section{Publisher's Note}

Springer Nature remains neutral with regard to jurisdictional claims in published maps and institutional affiliations.
Ready to submit your research? Choose BMC and benefit from:

- fast, convenient online submission

- thorough peer review by experienced researchers in your field

- rapid publication on acceptance

- support for research data, including large and complex data types

- gold Open Access which fosters wider collaboration and increased citations

- maximum visibility for your research: over 100M website views per year

At BMC, research is always in progress.

Learn more biomedcentral.com/submissions 